\title{
An innovative device for bobbin thread consumption measurement on industrial lockstitch sewing machines
}

\author{
Helder Carvalho \\ University of Minho \\ Dep.Textile Engineering \\ Campus de Azurém \\ 4800-058 Guimarães, \\ Portugal \\ helder@det.uminho.pt
}

\author{
Ana Rocha \\ University of Minho \\ Dep.Textile Engineering \\ Campus de Azurém \\ 4800-058 Guimarães, \\ Portugal \\ amrocha@det.uminho.pt
}

\author{
João L. Monteiro \\ University of Minho \\ Dep.Industrial Electronics \\ Campus de Azurém \\ 4800-058 Guimarães, \\ Portugal
}

monteiro@dei.uminho.pt

\author{
Luís F.Silva \\ University of Minho \\ Dep.Mechanical Engineering \\ Campus de Azurém \\ 4800 058 Guimarães, \\ Portugal
}

lffsilva@dem.uminho.pt

Abstract - This paper presents some development work following up on previous research on monitoring and control techniques for high-speed industrial sewing machines. The main objective of the studies is to develop techniques and devices to allow a faster set-up, greater flexibility and higher product quality in industrial sewing operations [1][2][3][4][6][7].

One of the main aspects of the sewing process is the interlacing of threads - stitch formation. It has been found that the on-line measurement of thread consumption can provide information about stitch adjustment and serve as a feedback variable for thread tension control[1][3]|4][7]. In this paper, an overview of this technique will be given, with particular focus on the quantification of bobbin thread consumption in lockstitch machines, one of the most widely used type of machine. In this specific case, measurement is difficult due to lack of physical space for the sensors.

Key Words: Sewing, stitch formation monitoring, tension control, seam quality, bobbin thread

\section{Introduction}

Although industrial sewing machines have been greatly improved with all kinds of technological developments since its first emergence, some characteristics of the materials that ate processed result in technical issues that are difficult to objectively manage and control.

The very unpredictable behaviour of the materials when they undergo the manufacturing operations require a subjective and empirical judgement in the machine set-up and general process design. This is made by producing samples and setting the machines on basis of trial-anderror, which is a time consuming operation with weak reproducibility. The machines are often equipped with complex devices to aid in material handling, but are blind regarding the process itself. They run with the initial set-up and are neither capable of adapting to varying sewing conditions nor can they detect anomalous operation. This means that both the correct setting of the machine as well

as control of the process and supervision of quality rely entirely on the skills of human operators.

The objective of the presented research project is to obtain methods and devices for a more complete and, in particular, more objective control of the process. This is an important requirement for the garment manufacturer that has to respond quickly and with quality to the market. Another emerging application is the increasing need to quantitatively monitor and even document sewing conditions concerning high-tech seams (air bags, safety belts, technical textiles), in the framework of quality certification.

The current paper presents the development of stitch formation monitoring methods relying on the measurement of thread consumptions. Previous work has shown that thread consumption can be used to asses the correct stitch adjustment. The following sections give a brief overview of the work developed so far, and a theoretical analysis of thread consumptions in sewing machines.

\section{The sewing test rig}

Figure 1 shows one of the two sewing test rigs developed to carry out research work in the parameterisation of sewing variables and determining their relation with seam quality.

The rig is composed of an overlock sewing machine equipped with sensors, signal conditioning hardware, and a PC with a data acquisition board. A software application developed in Labview provides extensive functions for acquisition, display, storage and analysis of all the sewing variables measured. 


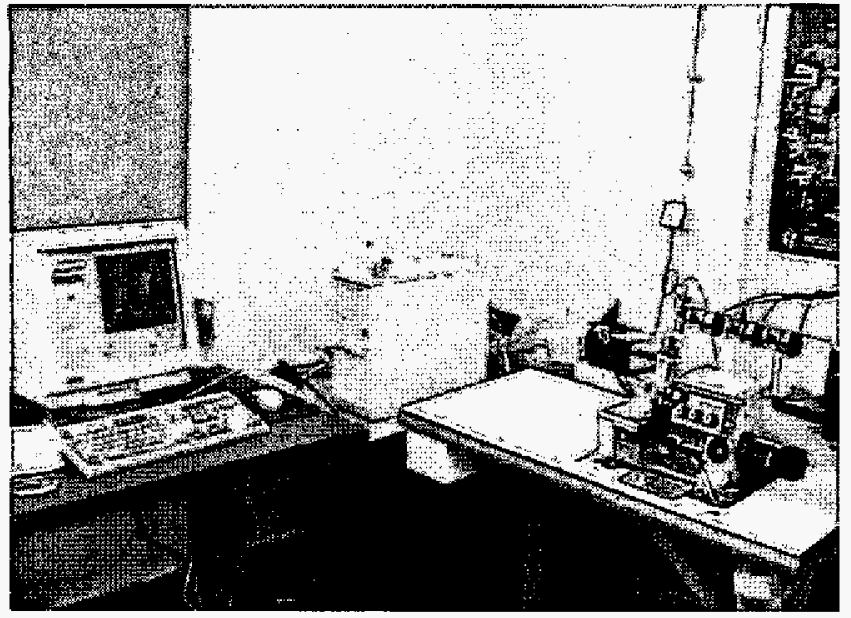

Fig.1: Overview of the sewing test rig

These can be grouped in three groups, relating to the phenomena under study:

- Needle penetration and withdrawal forces- measured by a piezoelectric sensor introduced in the needle-bar. A detailed description of this measurement can be found in [1][5]

- Behaviour of the feeding system- described by the measurement of force on and vertical displacement of the presser-foot's, measured by a piezoelectric sensor and an LVDT, respectively (Fig.3). Further information can be found in [2][6].

- Stitch formation - the interlacing of threads is described by measurement of thread tensions occurring during the stitch cycle and by the measurement of thread consumption. Figures 2 and 3 show the set-up for these measurements on the overlock machine. Previous work described in[1][3].

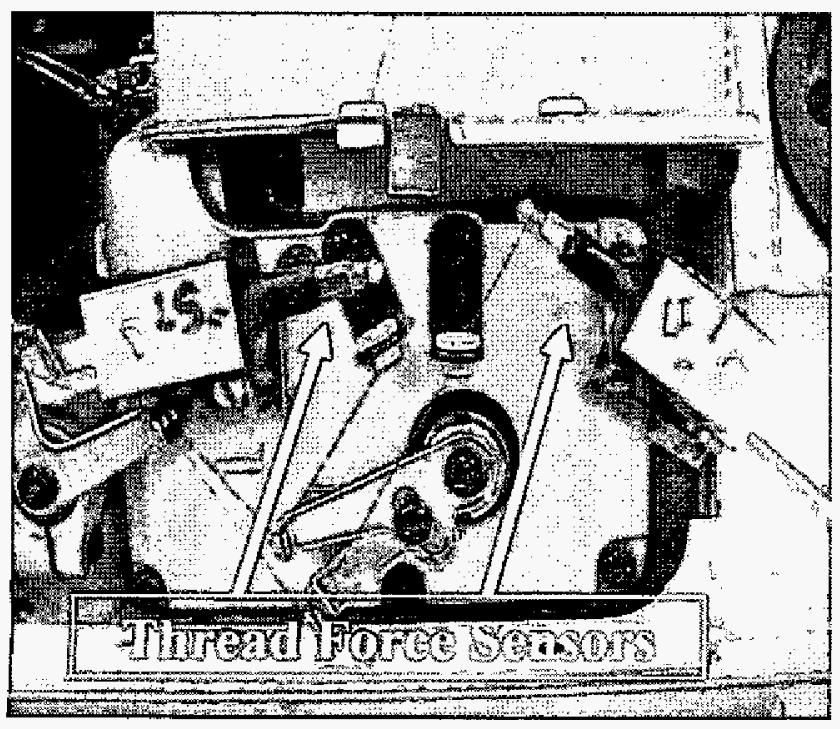

Fig.2: Thread force sensors: Cantilever beam with semiconductor strain gauges

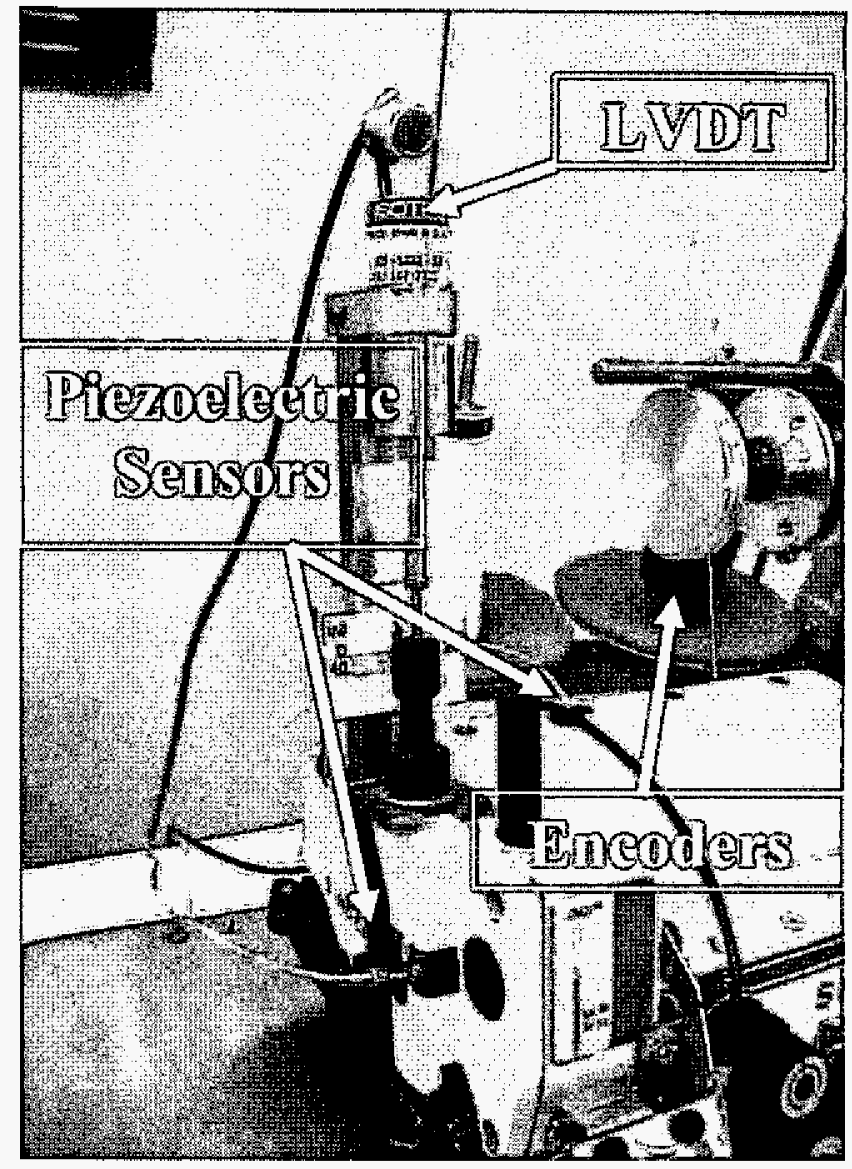

Fig 3: Measurement of presser-foot force and displacement, needle-bar force and thread consumption (only one thread shown)

\section{Thread consumption and its relation to tension adjustment}

In a sewing machine, stitch formation occurs by the synchronised movement of the needle, feeding system and other stitch formation elements. In this paper, two distinct cases will be analysed: the 504 overlock stitch (a chainstitch) and the 301 lockstitch. In the case of the 504 stitch, one needle and two loopers are used, each of them carrying a thread. The stitch formed by the machine is classified as type 504. Its schematic representation can be observed in Fig.4.

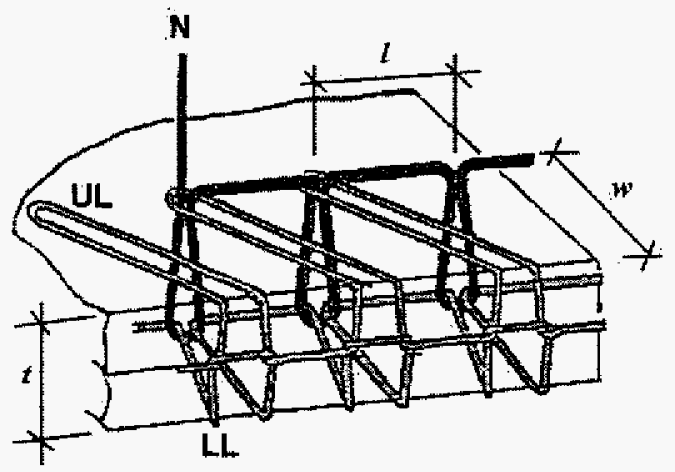

Fig.4: Threc-thread overlock stitch type 504 (N: Needle thread, LL: Lower Looper thread, UL: Upper Looper Thread;) 
The 301 lockstitch uses a needle and a bobbin, which is winded with thread and then inserted into a bobbin case. The stitch formation is quite different from chainstitches because instead of interlacing loops of threads, the threads are interlaced directly. Fig.5 shows the schematic representation of the lockstitch.

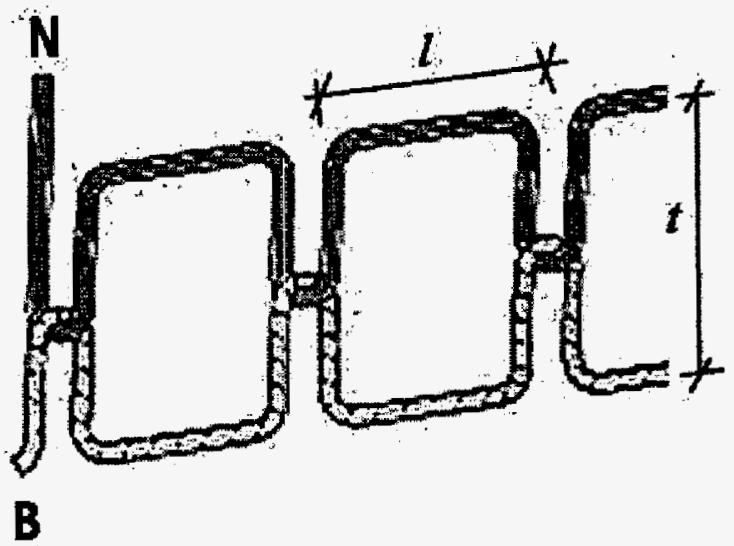

Fig.5: Lockstitch type 301 (N: Ncedle thread, B: Bobbin thread)

Thread consumption is directly related to the presented stitch geometries, and can be easily computed for each thread using the parameters

$$
\begin{array}{ll}
l: & \text { stitch length } \\
t: & \text { material thickness } \\
w: & \text { seam width (applicable to } 504 \text { stitch only) }
\end{array}
$$

Seam width is a constant for a particular machine, whilst stitch length is a parameter that can be adjusted at the machine. Material thickness depends on the material type and compression state. It can be measured, for a specific presser-foot compression force, by a specifically designed software module that uses the LVDT [7].

A correctly adjusted stitch should comply with the theoretical values of thread consumption. The correct balance and tensioning of the stitch are essential for a good performance of the seam, as well as for aesthetical appeal.

Previous studies have shown that both balance as well as the overall amount of tension of a stitch can be assessed by the comparison between actual and theoretical consumption values[1][3]. Fig.6 shows a comparison of a balanced (solid) with an unbalanced 504 stitch (dithered). Thread consumption has been measured in 10 seams for each situation; theoretical consumption is indicated by the transparent bars at the right of the measurements. As can be observed, an unbalance between the two looper threads has occurred.

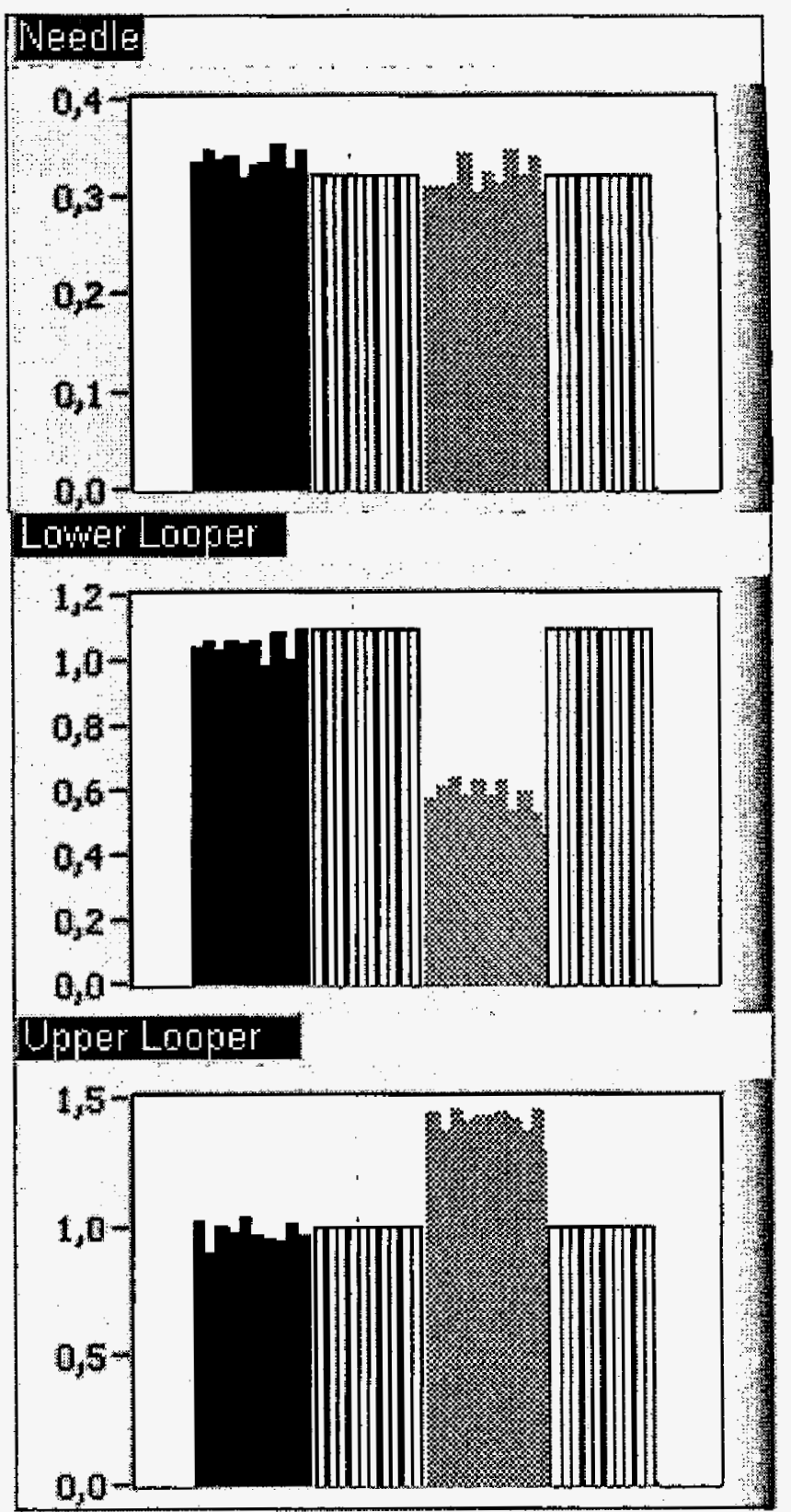

Fig.6: A verage thread consumption per stitch[cm], unbalanced versus balanced stitch

\section{Thread consumption measurement on lockstitch machines}

Measurement of needle thread consumption on the lockstitch machine can be achieved in the same way as it is done in the lockstitch machine. The needle thread is fed from a reel and it is easy to introduce an encoder in the thread path to count thread consumption.

However, this set-up is not possible for the bobbin thread. In this case, the physical space between the thread output point at the bobbin case, and the thread interlacing point, is too narrow to allow the introduction of any sensor. This can be observed in fig. 7 , which shows a photograph of the hook, where the bobbin case is inserted. 


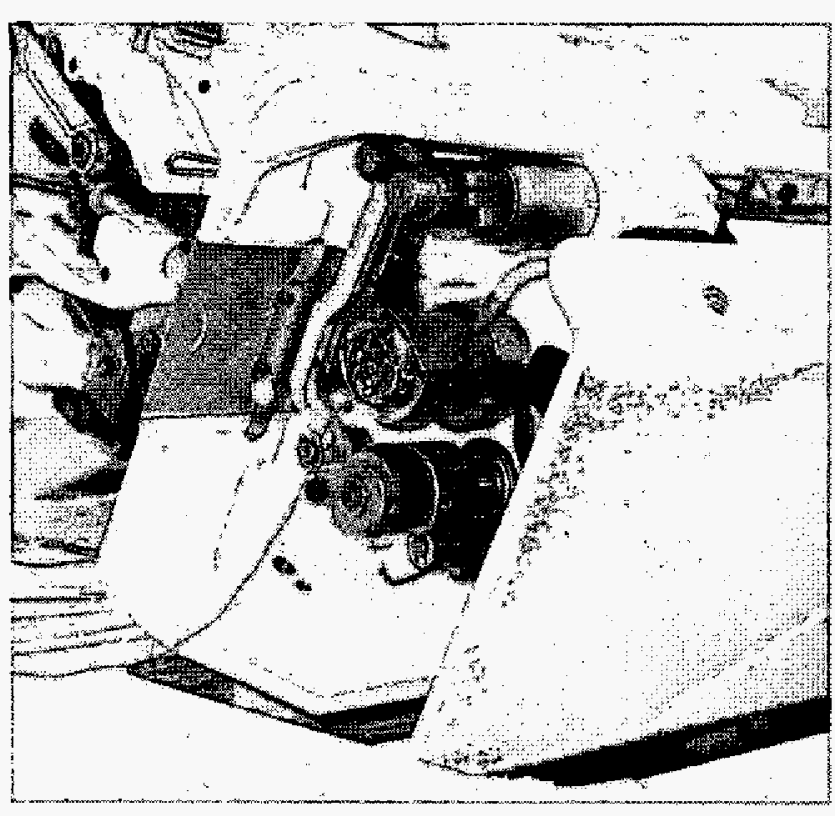

Fig.7: Lowcr stitch formation elements: Hook with inserted bobbin case

To overcome this limitation, an indirect measurement method has been devised. The quantification of bobbin thread consumption is made in two phases:

- Determine the bobbin thread winding characteristic

- Determine the unwinding of the bobbin thread

The following sections describe this process.

\subsection{Determination of bobbin winding characteristic}

The winding of thread to the bobbins is normally made on the machine itself, with a winding device that runs synchronously with the stitch formation process, as shown in fig.8.

The objective of this step is the determination of the following relation:

$$
l=f(\alpha)
$$

in which

$$
\begin{array}{ll}
l: & \text { length of winded thread } \\
\alpha . & \text { rotation angle of the bobbin }
\end{array}
$$

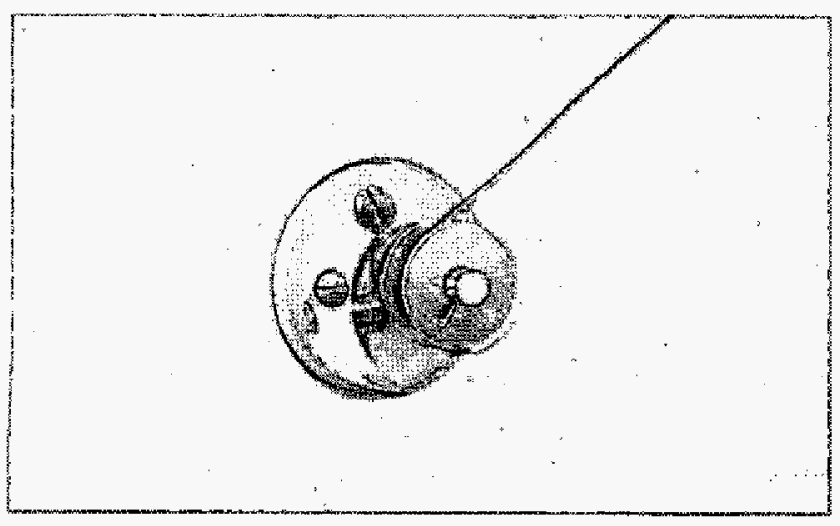

Fig.8: Thread winding device
This relation is variable, depending on the thread's thickness and on the tension with which it is winded on the bobbin.

To accomplish this measurement, an encoder is inserted into the thread path of the bobbin's winding thread. During the winding process, a counter counts the pulses produced at the encoder by the thread's motion.

The counting process uses a buffer that accumulates count values. A count value is pushed into the buffer at each stitch cycle. This is controlled by hardware, using a signal provided by the machine's motor that delivers a pulse at each rotation of the machine.

An appropriate calibration procedure is performed using a software module developed in previous work. This procedure is performed before the whole process (and only one time), and enables the conversion of count values into thread length. Using the relation between machine rotation and bobbin rotation, the length winded for each rotation of the bobbin is obtained by interpolation.

The resolution of this measurement can be increased, if necessary, producing more than one count value per rotation of the machine. This is possible using another signal provided by the machine's motor that delivers 256 pulses per rotation; a divide by two counter may be used to reduce the number of measurements to an adequate value.

\subsection{Determination of bobbin thread unwinding}

To obtain the bobbin thread consumed, the bobbin's rotation during the sewing process has to be detected. The bobbin's rotation is not mechanically synchronised with the needle movement. Rather, it is produced by the thread that is pulled out of the bobbin during the stitch formation process.

Establishing a physical connection to the bobbin, in order to detect its rotation, is mechanically not possible on lockstitch machines. To solve this problen, the-bobbin was marked with a pattern which divides it in 16 radial sections, being these sections alternately contrasting in black and white (fig.9).

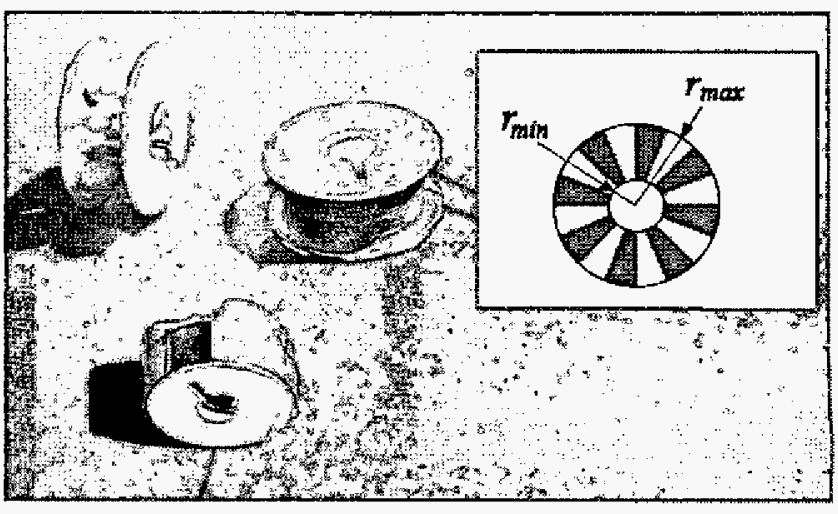

Fig.9: Bobbin, bobbin casc, pattern for optical rotation detection 
An optical sensor of the diffuse reflective type is then used to determine the bobbin's rotation angle. This type of sensor emits a light beam that is reflected by an object and detected by the sensor's receiver.

The selection of a sensor and the resolution of this measurement depend on several issues.

The resolution of the measurement depends in the first place on the number of sections marked on the bobbin. Specifically, the measurement resolution is worst when the bobbin is full. At this point, it can be quantified as :

$$
\operatorname{Res}=\frac{2 \pi r_{\max }}{N / 2}
$$

in which

Res: Measurement resolution

$r_{\text {max }}:$ maximum bobbin winding radius

Resolution improves with the number of sections. However, this number is limited by the characteristics of the optical sensor.

The spot diameter is the first parameter limiting the width of the marked sections. It determines the diameter of the light beam projected. Assuming that the optical detection is made at the center of the bobbin's radial sections, the maximum spot diameter related to the number of section by the following relation:

$$
d_{\max }=\frac{2 \pi \frac{r}{2}}{N}
$$

in which

$$
\begin{array}{ll}
d_{\text {max }}: & \text { maximum spot diameter } \\
r: & \text { bobbin radius } \\
N: & \text { number of sections }
\end{array}
$$

The second limitation results from the sensor's response time. The sensor has to be fast enough to respond to the black-white transitions when the bobbin is rotating at its maximum expected speed. To determine this speed, the following calculations are made.

Bobbin thread consumption per stitch in lockstitch is expressed by the following relation:

$$
C_{s}=l+t
$$

in which

$$
\begin{array}{ll}
C_{s}: & \text { Consumption per stitch } \\
l: & \text { stitch length } \\
t: & \text { material thickness }
\end{array}
$$

The unwinding of bobbin thread causes a rotation to the bobbin. The maximum rotational speed is reached when the thread content of the bobbin is at its lowest. At this point, the rotational speed is

$$
\omega_{\max }=v_{\max } \frac{C_{s}}{2 \pi r_{\min }}
$$

in which

$$
\begin{array}{ll}
\omega_{\max }: & \text { Maximum bobbin rotational speed [rps] } \\
v_{\max }: & \text { maximum sewing speed [stitches/s] } \\
C_{s}: & \text { bobbin thread consumption per stitch } \\
r_{\min }: & \text { minimum bobbin winding radius }
\end{array}
$$

The sensor's response time has thus to be faster than

$$
t_{r} \leq \frac{1}{N \omega \max }
$$

in which

$$
\begin{array}{ll}
t_{r}: & \text { Sensor response time } \\
N: & \text { number of sections }
\end{array}
$$

The calculations for the current case are presented in the next lines.

In a worst-case situation, the sewing machine would be sewing with a stitch length $l=5 \mathrm{~mm}$ and joining 3 plies of fabric with thickness $t=1.5 \mathrm{~mm}$. This results in a thread consumption per stitch of $C_{s}=9.5 \mathrm{~mm}$.

The minimum winding radius of the bobbin is $r_{\min }=3 \mathrm{~mm}$. Maximum speed of the sewing machine is $v_{\text {max }}=6000$ stitches/minute $=100$ stitches/s. In these conditions, maximum rotational speed of the bobbin is $\omega_{\max }=50.4 \mathrm{~Hz}$.

A review of economic optical sensors available on the market, which would fit into this device led the team to choose a diffuse reflective sensor that features a response time of $1 \mathrm{~ms}$ and a spot diameter of $2.5 \mathrm{~mm}$ at a range of about $10 \mathrm{~cm}$ (a convenient distance for application on the machine). Based on these values, the bobbin was marked with 16 sections. This results in a maximum spot diameter of $d_{m a x}=4.1 \mathrm{~mm}$ and a maximum response time of $t_{r}=1.24$ ms. The resolution of the measurement results in Res $=8.24 \mathrm{~mm}$. which is perfectly adequate for the application in hand. However, the performance of the overall measurement system is limited by the resolution of the bobbin winding characterisation, which can also be improved, if necessary, as explained previously. The current resolution is sufficient to allow the measurement of consumption in a complete seam. A stitch-by-stitch estimation is obtained by interpolation.

\footnotetext{
' $\mathrm{N}$ is divided by two because the counters only count high-to-low or low-to-high transitions, not both
} 


\subsection{Auxiliary functions}

The prototype implemented with the $\mathrm{PC}$, as well as a future dedicated device, need several auxiliary functions to assure reliable operation.

The first condition to be fulfilled is that the measurement has to be running whenever the machine is used, otherwise the process is enroneous until the end of the current bobbin. To achieve this, the motor is locked whenever the software is not running. This is achieved using a specific function of the motor, controlled by a digital output of the data acquisition board and a simple hardware interface.

The change of bobbin in the machine has to be indicated by the user. This will lead to the reset of the process, with the new winding information for the full bobbin.

During winding, the system should detect when the bobbin is full. The winding device uses a mechanical system that stops winding when the thread has reached a level (this level is adjusted mechanically). Considering that the length of thread is not known in advance, it is not possible to the software to stop the count process based on the winded thread. Instead, the software monitors the counted values and stops the process when values do not change for a certain time. This is the most economical solution, a more direct method would use a sensor to detect the state of the winding device.

In a practical system, it would be of advantage to use a bobbin identification system that could detect bobbin change automatically. This would be especially interesting for application in seams requiring maximum quality assurance, for safety reasons.

All this information has finally to be adequately made available in the user interface, allowing a quick and straightforward interaction with the user.

\section{Conclusions}

The proposed device is being tested using a test setup, in which the bobbin is wound on the machine's winding device and unwound manually. The thread length values provided by the software have so far been found to be very accurate, and the end of thread is also indicated with great precision. The optical detection had been previously successfully tested using a motor spinning the bobbin at about $77 \mathrm{rps}$. At this speed, the light spot has to be moved a little closer to the perimeter of the bobbin. A degradation of this performance is expected when the sensor is actually applied to the machine, considering the worse lighting conditions and dirt resulting from the sewing operation. In any case, the device is expected to perform well in normal sewing conditions, where rotational speed is significantly less than in the worst-case situation depicted.

Currently, the auxiliary functions of the system, including the user interface, are in a final stage of development. The device is expected to be an important component for the monitoring and control devices proposed.

\section{Acknowledgements}

The authors are grateful to FCT (Fundação para a Ciência e Tecnologia), project funding POSI/SRI/38944/2001.

\section{References}

[1] Carvalho, H., Optimization and Control of Processes in Apparel Manufactoring, $P h D$ thesis, University of Minho, Portugal, 2004.

[2] Silva, L. F., Estudo de Mecanismos Alternativos de Controlo do Sistema de Alimentação de Máquinas de Costura Industriais, $P h D$ thesis, University of Minho, Portugal. 2002

[3] Carvalho, Miguel, Estudo das relações entre os parâmetros de controlo, propriedades dos materiais e condiçōes de regulação numa máquina de costura corta-e-cose $P h D$ thesis, University of Minho, Portugal. 2002

[4] H. Carvalho, A.M. Rocha, J. Monteiro, Process Control in Next-Generation Sewing Machines - A Project Overview, Proceedings of the International Conference on Mechatronics (ICOM 2003), pp 203-208, Intemational Engineering Publishing, ISBN 1-86058-420-9, Loughborough, UK, 19-20 June 2003

[5] H.Carvalho, P.Ferreira, A.M.Rocha, J.Monteiro, Signal Feature Extraction for Sewing Analysis using Non-Linear Techniques, Proceedings of the 2003 IEEE International Symposium on Industrial Electronics (ISIE), IEEE, ISBN 0-7803-7912-8, Rio de Janeiro, Brasil, June 2003

[6] L.F.Silva, M. Lima, H. Carvalho, A.M.Rocha, F.N.Ferreira, J.Monteiro, C.Couto, Actuation, Monitoring and Control of Fabrics Feeding during High-Speed Sewing, Proceedings of the International Conference on Mechaironics (ICOM 2003), pp 471-476, International Engineering Publishing, ISBN 1-86058-420-9, Loughborough, UK, 19-20 June 2003

[7] H. Carvalho, A.M.Rocha, J.Monteiro L.F.Silva, Planning, Control and Monitoring Tools for Industrial Gamnent Assembly Processes, Proceedings of the International Conference on Industrial Technology, pp 381-386, IEEE, ISBN 0-7803-7852-0/03, Maribor, Slovenia, 10-12 December 2003 